\title{
ESTABILIDADE DIMENSIONAL E RESISTÊNCIA À TRAÇÃO PERPENDICULAR DE PAINÉIS FABRICADOS COM PARTÍCULAS TERMORRETIFICADAS ORIUNDAS DE EMBALAGENS DE Pinus sp. ${ }^{1}$
}

Benedito Rocha Vital ${ }^{2}$, Paulo Ivan Lima Andrade ${ }^{3}$, Angélica de Cássia Oliveira Carneiro², Carla Priscilla Távora $\mathrm{Cabral}^{3}$ e Ana Márcia Macêdo Carvalho²

\begin{abstract}
RESUMO - O objetivo deste trabalho foi avaliar o efeito do tratamento térmico em algumas propriedades de painéis de partículas, produzidos com resíduos de embalagens de Pinus sp. Foi utilizado o adesivo à base de ureia-formaldeído, na quantidade de $8 \%$. O experimento foi desenvolvido em um fatorial $4 \times 3 \times 3$, sendo quatro proporções de partículas $(25,50,75$ e $100 \%)$, termorretificadas, três temperaturas de tratamento térmico $\left(180,200\right.$ e $\left.220^{\circ} \mathrm{C}\right)$ e três repetições, totalizando 36 painéis. Foram produzidos mais três painéis com partículas sem tratamento térmico (testemunhas), totalizando 39 painéis. A absorção de água, inchamento em espessura e resistência à tração perpendicular foram determinadas de acordo com a Norma ABNT/NBR 14810-3 (2002). Os resultados desses testes foram comparados com os valores estabelecidos nas Normas ABNT/NBR 14810-3 (2002) e DIN 68761 (1) (1961). A estabilidade dimensional dos painéis aumentou com a adição de partículas termorretificadas, enquanto as propriedades mecânicas foram reduzidas. O efeito da adição de partículas termorretificadas nas propriedades dos painéis é maior à medida que se utilizam maiores temperaturas de tratamento térmico.

Palavras-chave: Tratamento térmico; Painéis de madeira aglomerada; Resíduos.

\section{DIMENSIONAL STABILITY AND RESISTANCE TO PERPENDICULAR TENSION OF BOARDS MADE WITH HEAT-TREATED PARTICLES FROM PACKAGINGS OF Pinus sp.}

\begin{abstract}
The objective of this study was to evaluate the effect of heat treatment on some properties of particle boards produced with Pinus sp. packing residues. Eight percent of urea-formaldehyde base adhesive was used. The experiment was conducted in a factorial $4 \times 3 \times 3$, being 4 proportions of heat-treated particles $(25,50,75$ and $100 \%)$, three temperatures of heat treatment $\left(180,200\right.$ and $\left.220^{\circ} \mathrm{C}\right)$ and three replications, totaling 36 boards. Three additional boards were made with particles without heat treatment (controls), totaling 39 boards. Water absorption, thickness swelling and resistance to perpendicular tension were determined according to ABNT/NBR 14810-3 (2002). The results of these tests were compared with the values established by ABNT/ NBR 14810-3 (2002) and DIN 68761 (1) (1961). The dimensional stability of the boards has increased with the addition of heat-treated particles, whereas mechanical properties were reduced. The effect of adding heat-treated particles on the properties of the boards was higher when using higher temperatures in heat treatment.
\end{abstract}

Keywords: Heat treatment, particleboard, residues.

\footnotetext{
${ }^{1}$ Recebido em 08.01.2014 aceito para publicação em 07.08.2014.

${ }^{2}$ Departamento de Engenharia Florestal, Universidade Federal de Viçosa, UFV, Brasil. E-mail: <bvital@ufv.br>, <cassiacarneiro@ufv.br> e<ana.marcia@ufv.br>.

${ }^{3}$ Programa de Pós-Graduação em Ciências Florestais, Universidade Federal de Viçosa, UFV, Brasil. E-mail: <paulo_gfx@hotmail.com> e $<$ lcajir@yahoo.com.br>.
} 


\section{INTRODUÇÃO}

Os painéis de madeira reconstituída são amplamente utilizados em mobiliário e na construção civil. Durante o processo produtivo desses painéis, é necessário que a madeira seja transformada em partículas, fazendo que a exigência em termos de qualidade da matériaprima não seja tão grande. Com isso, torna-se possível a utilização de resíduos. Sob essa perspectiva, a indústria de painéis de madeira reconstituída ocupa posição estratégica no cenário geopolítico, uma vez que as políticas públicas no Brasil e no mundo têm-se desenvolvido no sentido de reduzir, reutilizar e reciclar aquilo que é descartado como resíduo, conforme estabelecido pela Lei $n^{\circ} 12.305 / 10$, segundo a qual fica instituída a Política Nacional de Resíduos Sólidos.

A maior parte da matéria-prima destinada à produção dos painéis de madeira reconstituída no Brasil advém de plantios florestais. Entretanto, a utilização de resíduos pode representar para a indústria redução no custo de produção. Dessa maneira, maiores investimentos poderiam ser direcionados à melhoria da estabilidade dimensional desses painéis, que geralmente apresentam alta absorção de água e inchamento em espessura.

Em parte, esses problemas ocorrem devido ao principal adesivo utilizado, ureia-formaldeído, que apresenta baixa resistência à umidade. Entretanto, propriedades como geometria das partículas, densidade e higroscopicidade da madeira também refletem significativamente na qualidade dos painéis.

O tratamento térmico da madeira é alternativa promissora na melhoria das suas propriedades tecnológicas. A ação da temperatura garante, entre outras vantagens, um produto com maior estabilidade dimensional e resistência à degradação biológica (STAMM et al., 1946; ARAÚJO et al., 2012).

A ação do calor promove modificações químicas diferenciadas nos constituintes fundamentais da madeira. Conforme Byrne e Nagle (1997), a madeira começa a ser degradada a temperaturas próximas de $200^{\circ} \mathrm{C}$, com o início da decomposição das hemiceluloses, componentes que mais contribuem para a higroscopicidade da madeira, por apresentarem elevada quantidade de grupamentos hidroxílicos e estrutura completamente amorfa.

A celulose, por sua vez, apresenta resistência moderada, em função da presença de regiões cristalinas. A lignina é o componente da madeira mais resistente à degradação térmica. Apesar disso, ocorrem modificações significativas em sua estrutura, que podem ser constatadas pelo aumento na concentração de compostos fenólicos na madeira tratada termicamente (ESTEVES et al., 2008).

Boonstra et al. (2006) avaliaram o efeito do prétratamento térmico de partículas de Picea abies e Pinus sylvestris sobre as propriedades de painéis de madeira reconstituída, utilizando vapor sob alta pressão num primeiro estágio e, posteriormente, aquecimento a 180 ${ }^{\circ} \mathrm{C}$ em atmosfera de N2. A estabilidade dimensional dos painéis aumentou, enquanto a ligação interna foi reduzida.

Apesar disso, Paul et al. (2006) verificaram que painéis do tipo OSB produzidos com partículas de Pinus sylvestris, tratadas a 220 e $240^{\circ} \mathrm{C}$ em um secador rotatório, tiveram redução significativa no inchamento em espessura sem, no entanto, afetar a ligação interna.

Diante desses resultados, observou-se que o efeito do tratamento térmico nas propriedades dos painéis pode ser bastante diferenciado. Em parte, isso se deve às características peculiares da madeira de cada espécie utilizada. Entretanto, as condições em que se realiza o tratamento térmico, como características da atmosfera (oxidante ou inerte, seca ou úmida) e a aplicação de pressão influem diretamente sobre as propriedades do produto final.

O objetivo deste trabalho foi avaliar o efeito do tratamento térmico nas propriedades de painéis de partículas produzidos com madeira proveniente de resíduos de embalagens de Pinus sp.

\section{MATERIAL E MÉTODOS}

Para a produção das partículas, foram utilizados resíduos madeireiros provenientes de embalagens do tipo caixas, que são constituídas por painéis compensados e madeira sólida de Pinus sp.

As embalagens foram desmontadas, removendo-se os pregos e grampos. Em seguida, o material foi cortado e processado em um picador de cavacos. Posteriormente, os cavacos foram reduzidos a partículas em moinho de martelo. Na sequência, as partículas passaram por um peneiramento manual, em peneiras com malha de $2,5 \mathrm{~mm}$, recolhendo-se a fração retida nessa malha. 
As partículas foram tratadas termicamente nas temperaturas de 180,200 e $220^{\circ} \mathrm{C}$ durante $15 \mathrm{~min}$, depois de atingida a temperatura desejada. O teor de umidade inicial das partículas foi de aproximadamente $12 \%$.

O tratamento térmico foi realizado em um torrificador rotatório que consiste de um cilindro rotatório, acionado por um motor elétrico e aquecimento a gás (GLP). A temperatura foi monitorada através de um termômetro analógico, localizado em uma das laterais do cilindro, e controlada, regulando-se a entrada de gás.

Foram determinadas as seguintes propriedades das partículas:

- Umidade de equilíbrio higroscópico (UEH) nas condições de temperatura de $20 \pm 3^{\circ} \mathrm{C}$ e $65 \pm 5 \%$ de umidade relativa.

- Densidade básica segundo a metodologia proposta por Vital (1984).

Perda de massa: as partículas foram pesadas antes e depois do tratamento térmico, descontando-se o teor de umidade após o equilíbrio.

Para a produção dos painéis, o teor de umidade das partículas foi ajustado para $3 \%$. Os painéis foram produzidos nas dimensões de $40 \times 40 \times 1,5 \mathrm{~cm}$, com densidade aparente desejada de $600 \mathrm{~kg} / \mathrm{m}^{3}$.

Foi utilizado o adesivo à base de ureia-formaldeído, na quantidade de $8 \%$ sobre a massa de partículas secas. Utilizou-se o catalisador sulfato de amônio, no teor de $1,5 \%$, sobre a quantidade de sólidos do adesivo.

As partículas termorretificadas foram misturadas a partículas não tratadas, nas proporções de 25,50 , 75 e $100 \%$. Foram produzidos também painéis com partículas sem tratamento térmico (testemunhas).

O adesivo foi aplicado nas partículas, empregando-se um encolador dotado de uma pistola pneumática. A prensagem foi realizada em prensa mecânica de pratos planos, a uma pressão de $32 \mathrm{kgf} / \mathrm{cm}^{2}$ e na temperatura de $180^{\circ} \mathrm{C}$, durante $8 \mathrm{~min}$.

As propriedades de absorção de água (AA), inchamento em espessura (IE) e resistência à tração perpendicular (TP) dos painéis, foram determinadas de acordo com a Norma ABNT/NBR 14810-3 (2002). Os corpos de prova foram climatizados até a umidade de equilíbrio em temperatura de $20 \pm 3{ }^{\circ} \mathrm{C}$ e $65 \pm 5 \%$ de umidade relativa, utilizando-se uma câmara climática.
A absorção de água e o inchamento em espessura foram comparados aos valores máximos estabelecidos pela Norma DIN 68761 (1) (1961). A resistência à tração perpendicular foi comparada com o valor requerido pela Norma ABNT/NBR 14810-3 (2002).

Avaliou-se a molhabilidade dos painéis, depositando uma gota de adesivo de aproximadamente $30 \mu \mathrm{L}$ sobre a superfície da amostra e utilizando uma seringa com capacidade de $1 \mathrm{~mL}$ e graduada em intervalos de 10 $\mu \mathrm{L}$, que foi posicionada a uma altura fixa de $8 \mathrm{~mm}$.

Após um período de $2 \mathrm{~min}$, obteve-se uma fotografia, com dimensões de $1280(\mathrm{H})$ x 1024 (V) pixels, utilizando o software Pixelink capture e uma câmera Pixelink, modelo PL-A662, acoplada a uma lupa estereoscópica ZEISS Stemi 2.000-C. O procedimento foi repetido 10 vezes para cada tratamento, sendo realizado em um ambiente com temperatura de $20 \pm 2{ }^{\circ} \mathrm{C}$ e $65 \pm 5 \%$ de umidade relativa. $\mathrm{O}$ ângulo de contato entre o adesivo e a superfície das amostras foi obtido utilizando o software AxioVision.

O experimento foi analisado segundo um delineamento inteiramente casualisado em um fatorial $4 \times 3 \times 3$, sendo quatro proporções de partículas termorretificadas $(25,50,75$ e $100 \%)$ adicionadas aos painéis, três temperaturas de tratamento $(180,200$ e $220^{\circ} \mathrm{C}$ ) e três repetições, totalizando 36 painéis. Foram produzidos três painéis com partículas sem tratamento térmico (testemunhas), perfazendo um total de 39 painéis.

Verificadas a normalidade dos dados e a homogeneidade da variância, o efeito dos tratamentos foi determinado através de análise de variância, empregando-se o software estatístico SAEG. Observadas as diferenças significativas, as médias foram comparadas entre si, utilizando o teste de Tukey a 95\% de significância. Para comparar as médias dos painéis produzidos com partículas termorretificadas com a testemunha, utilizou-se o teste de Dunnett a 95\% de significância.

\section{RESULTADOS}

A maior rugosidade da superfície, normalmente observada em painéis de madeira, e a superfície da madeira sólida, bem como os espaços vazios entre as partículas, dificultaram a mensuração do ângulo de contato. Não obstante, observou-se que o ângulo de contato médio dos painéis fabricados com partículas não termorretificas foi igual a $57^{\circ}$. Nos painéis fabricados

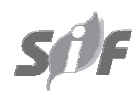

Revista Árvore, Viçosa-MG, v.38, n.5, p.951-959, 2014 
com partículas termorretificadas, observaram-se ângulos de contato médios iguais a 62,66 e $71^{\circ}$, para termorretificação aos 180,200 e $220^{\circ} \mathrm{C}$, respectivamente.

A Tabela 1 contém os valores médios de umidade de equilíbrio higroscópico (UEH), perda de massa e densidade básica das partículas, em função da temperatura do tratamento térmico. Observa-se, nessa tabela, que houve redução da umidade de equilíbrio à medida que aumentou a temperatura de tratamento das partículas.

A Tabela 2 contém os valores médios de absorção de água (AA), o inchamento em espessura (IE) após $2 \mathrm{~h}$ de imersão e a resistência à tração perpendicular
(TP) da testemunha e dos painéis produzidos com partículas termorretificadas. Nem a proporção nem a temperatura de torreifação afetaram o teor de água adsorvida. Verifica-se, também na Tabela 2, que os painéis produzidos com $75 \%$ de partículas termorretificadas incharam, aproximadamente, 30\% menos que a testemunha. Nas chapas contendo apenas partículas termorretificadas, o inchamento em espessura chegou a ser reduzido pela metade. A utilização de partículas tratadas nas temperaturas de 200 e $220^{\circ} \mathrm{C}$ também proporcionou resultados semelhantes. Contudo, a resistência à tração perpendicular das chapas, contendo partículas termorretificadas, foi significativamente inferior à resistência da testemunha.

Tabela 1 - Umidade de equilíbrio higroscópico (UEH), perda e massa e densidade básica das partículas de Pinus sp., em função da temperatura de torrefação.

Table 1 - Equilibrium moisture content (EMC), weight loss and basic density of the Pinus sp. particles, according to the heat treatment temperatures.

\begin{tabular}{cccc}
\hline Temperatura $\left({ }^{\circ} \mathrm{C}\right)$ & UEH $(\%)$ & Perda de massa $(\%)$ & Densidade básica $\left(\mathrm{g} / \mathrm{cm}^{3}\right)$ \\
\hline Testemunha & $10,55 \mathrm{~A}$ & $0,00 \mathrm{C}$ & $0,35 \mathrm{~B}$ \\
180 & $5,32 \mathrm{~B}$ & $3,37 \mathrm{~B}$ & $0,38 \mathrm{AB}$ \\
200 & $4,55 \mathrm{C}$ & $5,24 \mathrm{~B}$ & $0,41 \mathrm{~A}$ \\
220 & $3,39 \mathrm{D}$ & $10,56 \mathrm{~A}$ & $0,41 \mathrm{~A}$ \\
\hline
\end{tabular}

Médias ao longo das colunas seguidas de mesmas letras maiúsculas não diferem significativamente entre si, pelo teste Tukey a $5 \%$ de probabilidade.

Averages along the columns followed by the same capital letters do not differ significantly among themselves by Tukey test a $5 \%$ probability.

Tabela 2 - Comparação entre as médias de absorção de água (AA), inchamento em espessura (IE) e resistência à tração perpendicular (TP) da testemunha e dos painéis produzidos com partículas termorretificadas, em função da proporção e da temperatura.

Table 2 - Comparison between water absorption (WA) means, thickness swelling (TS) and resistance to perpendicular tension (RPT) of control and the boards produced with heat-treated particles, according to the proportion and temperature.

\begin{tabular}{|c|c|c|c|c|c|}
\hline \multirow[t]{2}{*}{ Proporção (\%) } & \multicolumn{2}{|c|}{ AA $(\%)$} & \multicolumn{2}{|c|}{ IE $(\%)$} & \multirow[t]{2}{*}{$\mathrm{TP}(\mathrm{MPa})$} \\
\hline & $2 \mathrm{~h}$ & $24 \mathrm{~h}$ & $2 \mathrm{~h}$ & $24 \mathrm{~h}$ & \\
\hline Testemunha & $80,1 \mathrm{~A}$ & $92,5 \mathrm{~A}$ & $26,5 \mathrm{~A}$ & $30,5 \mathrm{~A}$ & $0,89 \mathrm{~A}$ \\
\hline 25 & $82,1 \mathrm{~A}$ & $94,4 \mathrm{~A}$ & $23,7 \mathrm{~A}$ & $28,1 \mathrm{~A}$ & $0,52 \mathrm{~B}$ \\
\hline 50 & $80,9 \mathrm{~A}$ & $92,2 \mathrm{~A}$ & $21,0 \mathrm{~A}$ & $25,3 \mathrm{~A}$ & $0,44 \mathrm{~B}$ \\
\hline 75 & $77,9 \mathrm{~A}$ & $88,8 \mathrm{~A}$ & $16,8 \mathrm{~B}$ & $20,6 \mathrm{~B}$ & $0,37 \mathrm{~B}$ \\
\hline 100 & $69,6 \mathrm{~A}$ & $85,9 \mathrm{~A}$ & $13,1 \mathrm{~B}$ & $17,2 \mathrm{~B}$ & $0,37 \mathrm{~B}$ \\
\hline Média & 78,1 & 90,7 & 20,2 & 24,4 & 0,52 \\
\hline \multicolumn{6}{|l|}{ Temperatura $\left({ }^{\circ} \mathrm{C}\right)$} \\
\hline Testemunha & $80,1 \mathrm{~A}$ & $92,5 \mathrm{~A}$ & $26,5 \mathrm{~A}$ & $30,5 \mathrm{~A}$ & $0,89 \mathrm{~A}$ \\
\hline 180 & $82,6 \mathrm{~A}$ & $94,4 \mathrm{~A}$ & $23,6 \mathrm{~A}$ & $28,4 \mathrm{~A}$ & $0,46 \mathrm{~B}$ \\
\hline 200 & $77,3 \mathrm{~A}$ & $90,7 \mathrm{~A}$ & $17,6 \mathrm{~B}$ & $22,0 \mathrm{~B}$ & $0,42 \mathrm{~B}$ \\
\hline 220 & $73,0 \mathrm{~A}$ & $85,8 \mathrm{~A}$ & $14,9 \mathrm{~B}$ & $18,1 \mathrm{~B}$ & $0,40 \mathrm{~B}$ \\
\hline Média & 77,8 & 90,5 & 19,3 & 23,4 & 0,46 \\
\hline
\end{tabular}

Médias ao longo das colunas seguidas de mesmas letras não diferem significativamente da testemunha, pelo teste de Dunnett a $5 \%$ de probabilidade. Averages along the columns followed by the same letter do not differ significantly from the control, by Dunnett's test at $5 \%$ probability.

Revista Árvore, Viçosa-MG, v.38, n.5, p.951-959, 2014 
Comparações entre as médias da absorção de água, após 2 e 24 h de imersão, e em função da temperatura e da proporção de partículas termorretificadas na composição dos painéis, excluída a testemunha, estão contidas nas Tabelas 3 e 4, respectivamente.

Na Tabela 2, apresenta-se, também, o efeito da proporção de partículas termorretificadas e da temperatura sobre a resistência à tração perpendicular dos painéis, contendo apenas partículas termorretificadas. As diferenças foram significativas. Tanto o aumento na temperatura de termorretificação quanto a proporção de partículas termorretificadas ocasionaram redução no teor de água absorvida e redução na resistência à tração perpendicular dos painéis.

Os valores médios de absorção de água, após 24 h de imersão, podem ser observados na Tabela 4. A Norma DIN 68761 (1) (1961) preconiza que a absorção não deve ser superior a 10 e $15 \%$ após 2 e 24 h de imersão, respectivamente. Portanto, nenhum dos painéis atendeu aos requisitos dessa norma.

A Tabela 5 contém as comparações entre as médias de inchamento em espessura, após a imersão em água durante 2 e 24 h, em função da proporção e temperatura de torreifação. Verifica-se, nessa tabela, que os painéis produzidos com $100 \%$ de partículas tratadas a $220^{\circ} \mathrm{C}$ atenderam à Norma DIN 68761 (1) (1961), que estabelece inchamento máximo em espessura após 2 h de imersão de $6 \%$. Nas demais condições, os painéis incharam mais que o máximo permitido por essa norma.

\section{DISCUSSÃO}

O aumento do ângulo de contato e a consequente redução na molhabilidade dos painéis indicam redução dos grupamentos hidroxílicos nas partículas tratadas
Tabela 4 - Valores médios de absorção de água (AA) após $24 \mathrm{~h}$ de imersão em água, em função da proporção de partículas termorretificadas e da temperatura de termorretificação.

Table 4-Mean values of water absorption (WA) after 24 hours of immersion in water, as affected by the proportion of heat-treated particles and heat treatment temperature.

\begin{tabular}{cc}
\hline Proporção $(\%)$ & AA $24 \mathrm{~h}(\%)$ \\
\hline 25 & $94,4 \mathrm{~A}$ \\
50 & $92,2 \mathrm{AB}$ \\
75 & $88,8 \mathrm{BC}$ \\
100 & $85,9 \mathrm{C}$ \\
\hline Temperatura $\left({ }^{\circ} \mathrm{C}\right)$ & \\
\hline 180 & $94,4 \mathrm{~A}$ \\
200 & $90,7 \mathrm{~A}$ \\
220 & $85,8 \mathrm{~B}$ \\
\hline
\end{tabular}

Médias seguidas de mesmas letras não diferem significativamente entre si, pelo teste de Tukey a $5 \%$ de probabilidade. Averaged over the same column followed by capital letters along the lines followed by the same letter do not differ significantly among themselves by Tukey test at 5\% probability.

termicamente, gerando uma superficie parcialmente inativada. Além disso, é provável que o tratamento térmico tenha alterado a estrutura dos componentes da parede celular, principalmente das polioses, além da plasticização da lignina, resultando na redução da molhabilidade dos painéis, conforme sugerido por Hakkou et al. (2005). Outrossim, Vernois (2001) observou que tratamentos térmicos acima de $200^{\circ} \mathrm{C}$ tornam a superfície da madeira parcialmente hidrofóbica, de maneira que a absorção de adesivos e vernizes fique mais lenta em relação à de uma madeira não tratada. Isso acontece porque a energia superficial da madeira é afetada pela temperatura, reduzindo a sua molhabilidade. Além disso, segundo Jennings (2003), a tensão superficial e a viscosidade do líquido, bem como a rugosidade superficial e a porosidade da madeira, são fatores que

Tabela 3 - Valores médios de absorção de água (\%), após 2 h de imersão em água, em função da temperatura e da proporção de partículas termorretificadas.

Table 3 - Mean values of water absorption (\%) after 2 hours of immersion in water, as affected by temperature and the proportion of heat-treated particles.

\begin{tabular}{cccc}
\hline Proporção $(\%)$ & \multicolumn{4}{c}{ Temperatura $\left({ }^{\circ} \mathrm{C}\right)$} \\
\cline { 2 - 5 } & 180 & 200 & 220 \\
\hline 25 & $81,67 \mathrm{Aa}$ & $81,70 \mathrm{Aa}$ & $82,81 \mathrm{Aa}$ \\
50 & $83,95 \mathrm{Aa}$ & $78,91 \mathrm{Aa}$ & $79,73 \mathrm{ABa}$ \\
75 & $81,21 \mathrm{Aa}$ & $78,24 \mathrm{Aba}$ & $74,20 \mathrm{Ba}$ \\
100 & $83,62 \mathrm{Aa}$ & $70,17 \mathrm{Bb}$ & $55,14 \mathrm{Cc}$ \\
\hline
\end{tabular}

Médias ao longo das colunas seguidas de mesmas letras maiúsculas e ao longo das linhas seguidas de mesmas letras minúsculas não diferem significativamente entre si, pelo teste de Tukey a 5\% de probabilidade.

Means followed by same letters do not differ significantly among themselves by Tukey test at $5 \%$ probability. 
Tabela 5 - Valores médios de inchamento em espessura (IE), após 2 e 24 h de imersão em água (\%), em função da proporção de partículas termorretificadas e da temperatura.

Table 5 - Mean values of thickness swelling (TS) after 2 and 24 hours of immersion in water (\%), as affected by heattreated particles proportion and temperature.

\begin{tabular}{ccccccc}
\hline Proporção (\%) & \multicolumn{5}{c}{ Temperatura $\left({ }^{\circ} \mathrm{C}\right)$} \\
\cline { 2 - 7 } & \multicolumn{2}{c}{180} & \multicolumn{2}{c}{200} & \multicolumn{2}{c}{220} \\
\cline { 2 - 7 } & IE $2 \mathrm{~h}(\%)$ & IE $24 \mathrm{~h} \mathrm{( \% )}$ & IE $2 \mathrm{~h}(\%)$ & IE $24 \mathrm{~h} \mathrm{( \% )}$ & IE $2 \mathrm{~h}(\%)$ & IE $24 \mathrm{~h}(\%)$ \\
\hline 25 & $24,42 \mathrm{ABa}$ & $28,54 \mathrm{ABab}$ & $23,88 \mathrm{Aa}$ & $30,01 \mathrm{Aa}$ & $22,92 \mathrm{Aa}$ & $25,9 \mathrm{Ab}$ \\
50 & $26,23 \mathrm{Aa}$ & $31,39 \mathrm{Aa}$ & $19,77 \mathrm{Bb}$ & $23,99 \mathrm{Bb}$ & $17,11 \mathrm{Bc}$ & $20,59 \mathrm{Bc}$ \\
75 & $21,04 \mathrm{Ca}$ & $26,01 \mathrm{Ba}$ & $15,71 \mathrm{Cb}$ & $19,45 \mathrm{Cb}$ & $13,76 \mathrm{Cb}$ & $16,29 \mathrm{Cc}$ \\
100 & $22,57 \mathrm{BCa}$ & $27,64 \mathrm{Ba}$ & $10,85 \mathrm{Db}$ & $14,51 \mathrm{Db}$ & $6,00 \mathrm{Dc}$ & $9,45 \mathrm{Dc}$ \\
\hline
\end{tabular}

Médias ao longo das colunas seguidas de mesmas letras maiúsculas e ao longo das linhas seguidas de mesmas letras minúsculas não diferem significativamente entre si, pelo teste de Tukey a $5 \%$ de probabilidade.

Averaged over the same column followed by capital letters along the lines followed by the same letter do not differ significantly among themselves by Tukey test at 5\% probability.

influenciam no ângulo de contato. A porosidade, por exemplo, impede que a gota se equilibre e mantenha sua conformação, permitindo a penetração do líquido na madeira (CÉSAR, 2011).

A redução da umidade de equilíbrio, à medida que se aumentou a temperatura de tratamento das partículas, também indica redução na quantidade de grupamentos OH disponíveis para adsorção de água (PAUL et al., 2006; ARAÚJO et al., 2012). Isso provavelmente é decorrente da degradação das polioses, que é o componente mais higroscópico da parede da célula lenhosa. Entretanto, a ocorrência de ligações cruzadas na lignina e a degradação das regiões amorfas da celulose também podem contribuir para a redução da higroscopicidade (TJEERDSMA et al., 1998).

A redução no teor de umidade de equilíbrio das partículas possibilita, em princípio, a produção de painéis com maior estabilidade dimensional. Entretanto, a degradação dos grupos hidrofílicos e a consequente redução na molhabilidade podem dificultar a ação do adesivo na consolidação do painel, uma vez que a adesão química é resultante do contato entre os grupos hidroxílicos da madeira e as moléculas do adesivo.

Segundo Girard e Shah (1991), a madeira começa a sofrer degradação térmica a partir de $180^{\circ} \mathrm{C}$, com a liberação de dióxido de carbono, ácido acético e alguns componentes fenólicos e a consequente perda de massa. Neste trabalho, as perdas de massa (Tabela 1) das partículas tratadas a 180 e $200{ }^{\circ} \mathrm{C}$ não diferiram entre si. No entanto, nas partículas aquecidas a $220^{\circ} \mathrm{C}$, observou-se perda de massa igual a $10,56 \%$, que foi significativamente superior ao verificado nas demais temperaturas. Os valores foram, também, numericamente superiores aos observados por Paul et al. (2006), que constataram perdas máximas de massa de $2 \%$ nas temperaturas de 180,200 e $220{ }^{\circ} \mathrm{C}$, ao realizarem o tratamento térmico de partículas de Pinus silvestris utilizando um secador rotatório em escala laboratorial. Entretanto, o processo foi realizado numa atmosfera com reduzido teor de oxigênio, e as partículas entraram no equipamento com um teor de umidade de $4 \%$. Dessa maneira, as variáveis do processo utilizadas por aqueles autores podem ter contribuído para a menor perda de massa.

Além da perda de massa, o tratamento térmico da madeira provoca redução no seu volume, o que pode ocasionar redução na densidade. Neste trabalho, à exceção das partículas tratadas a $180^{\circ} \mathrm{C}$, todas as demais apresentaram maior densidade que as não tratadas. Isso indica que a contração volumétrica da madeira foi maior do que a sua perda de massa.

Com diminuição dos grupamentos $\mathrm{OH}$ disponíveis para adsorção das moléculas de água, pode ocorrer aproximação das microfibrilas de celulose após o tratamento térmico, o que resulta na contração volumétrica da madeira (VITAL; TRUGILHO, 1997).

A absorção de água dos painéis produzidos com partículas termorretificadas não diferiu significativamente da testemunha, isso provavelmente se deve ao fato de os adesivos à base de ureia-formaldeído se degradarem quando em contato com a água. Dessa maneira, mesmo com a utilização de partículas menos higroscópicas, não houve redução significativa na absorção de água dos painéis fabricados com partículas termorretificadas quando comparadas com a testemunha. No entanto, excluída a testemunha e analisando o efeito da temperatura 
e da proporção de partículas termorretificadas na composição dos painéis, observou-se que aqueles produzidos apenas com partículas tratadas a $220{ }^{\circ} \mathrm{C}$ absorveram significativamente menos água em relação aos demais (Tabelas 3 e 4). Reduções significativas na absorção de água também foram obtidas por Mendes (2010), ao produzir painéis do tipo OSB com partículas tratadas a 200 e $240{ }^{\circ} \mathrm{C}$.

Possivelmente, devido à redução nos grupamentos hidroxílicos, as partículas termorretificadas tiveram menor percentual de água de adesão, reduzindo a absorção de água pelo painel. Comparando o efeito dos tratamentos apenas sobre chapas fabricadas com partículas termorretificadas (Tabela 5), observa-se que tanto a proporção de partículas termorretificadas quanto a temperatura de termorretificação interferiram no inchamento em espessura, porém não afetaram significativamente a absorção de água. De modo geral, o aumento da temperatura, bem como o incremento na quantidade de partículas termorretificadas, resultou na redução do inchamento em espessura. Apenas nos painéis produzidos com partículas tratadas a $180^{\circ} \mathrm{C}$ não se observou essa tendência. Melhor estabilidade de chapas fabricadas com partículas termorretificadas foram também percebidas por Mendes (2010) e por Paul et al. (2006). Esses resultados indicaram que, mesmo com a utilização de um adesivo não resistente à água, a incorporação de partículas termorretificadas nos painéis pode aumentar significativamente a estabilidade dimensional, conforme foi observado, também, por Goroyias e Hale (2002), que estudaram o efeito do tratamento térmico de partículas strand nas temperaturas de $200,210,220,230,240,250$ e $260^{\circ} \mathrm{C}$, por um período de $20 \mathrm{~min}$.

De acordo com Kelly (1977), o inchamento em espessura dos painéis de madeira reconstituída ocorre devido à liberação das tensões de compressão oriundas do processo de prensagem e, também, devido ao inchamento das partículas de madeira.

Possivelmente, as partículas submetidas ao tratamento apresentaram menor inchamento, em decorrência da redução dos grupamentos hidroxílicos disponíveis para adsorção da água nos constituintes da parede celular. Além disso, com o aumento da densidade das partículas termorretificadas, menor quantidade destas foi necessária durante a formação do colchão para obter a densidade predeterminada do painel. Dessa maneira, a taxa de compactação, as tensões de compressão foram reduzidas e, consequentemente, os painéis apresentaram menor inchamento em espessura.

Possivelmente, a redução na higroscopicidade das partículas termorretificadas fez que elas absorvessem menos água, resultando em menor inchamento. Aliado a esse fato, os painéis com partículas termorretificadas tiveram redução nas tensões de compressão devido à menor taxa de compactação, que, por sua vez, está associada à maior densidade das partículas tratadas termicamente.

A resistência à tração perpendicular, também conhecida como ligação interna, é importante parâmetro na avaliação da qualidade de um painel, pois está diretamente associada à adesão das partículas de madeira. Os resíduos de embalagens de madeira utilizados para produção das partículas, por terem sido gerados de compensado, eram compostos de adesivo polimerizado, o que poderia afetar a ligação interna dos painéis. Contudo, pode-se observar na Tabela 2 que a testemunha apresentou resistência à tração perpendicular igual a 0,89 Mpa, valor similar ao obtido por Iwakiri et al. (2001) em painéis de partículas fabricados com mistura de cinco espécies de pinus, obtendo resistência à tração perpendicular média igual a $0,85 \mathrm{Mpa}$. Dessa maneira, conclui-se que o resíduo de adesivo polimerizado na matéria-prima não afetou a adesão entre as partículas.

Observa-se, contudo, na Tabela 2 que, apesar de o tratamento térmico melhorar a estabilidade dimensional dos painéis, a utilização de partículas termorretificadas reduziu entre 40 e $60 \%$ a resistência à tração perpendicular dos painéis, em comparação com a testemunha. Resultados semelhantes também foram observados por Boonstra et al. (2006) e Mendes (2010), ao produzirem painéis do tipo OSB utilizando partículas termorretificadas. Entretanto, Paul et al. (2006) e Mendes (2010) não observaram diferença significativa de resistência à tração perpendicular em painéis do tipo OSB produzidos com partículas termorretificadas. Entretanto, vale destacar que esses autores utilizaram adesivos à base de melamina-ureia-fenolformaldeído e fenolformaldeído, que formam ligações cruzadas durante a polimerização do adesivo, aumentando a resistência da linha de cola (PIZZI; MITTAL, 1994).

Sernek et al. (2004) afirmaram que o tratamento térmico das partículas acima de $180^{\circ} \mathrm{C}$ pode reduzir a resistência à tração perpendicular dos painéis, devido

Revista Árvore, Viçosa-MG, v.38, n.5, p.951-959, 2014 
à movimentação dos extrativos para a superfície das partículas e, também, à inativação parcial dessa superfície. Isso ocasiona diminuição dos grupamentos $\mathrm{OH}$ disponíveis, acarretando redução na adsorção química entre a madeira e o adesivo. Além disso, a condensação de componentes produzidos pela degradação térmica da madeira na superfície das partículas, como o alcatrão, pode ter reduzido a permeabilidade da superfície, prejudicando a adesão.

Considerando apenas os painéis contendo partículas termorretificadas, a maior resistência $(0,52$ Mpa) foi observada nos painéis contendo $25 \%$ dessas partículas. A resistência dos painéis contendo 50\% de partículas termorretificadas foi igual a 0,44 Mpa, valor significativamente inferior ao anterior. Nas proporções de 75 e $100 \%$, obtiveram-se resistências iguais a $0,37 \%$, valor significativamente inferior aos observados nas proporções iguais a 25 e $50 \%$.

Aumento na temperatura de termorretificação de 180 para 200 e $220^{\circ} \mathrm{C}$ ocasionou perda significativa de resistência à tração perpendicular $(0,46 ; 0,42$; e 0,40 Mpa, respectivamente). Contudo, apesar da redução da resistência à tração perpendicular, todos os painéis produzidos com partículas termorretificadas atenderam à exigência da Norma ABNT/NBR 14810-2 (2002), que estabelece um valor mínimo de $0,35 \mathrm{MPa}$ para painéis de média densidade.

\section{CONCLUSÃO}

A termorretificação das partículas é um tratamento que reduz tanto a adsorção quanto a absorção de água, melhorando a estabilidade dimensional dos painéis.

As propriedades mecânicas podem ser reduzidas.

O efeito da adição de partículas termorretificadas nas propriedades físicas e de resistência à tração perpendicular dos painéis foi maior quando a termorretificação ocorreu acima de $200{ }^{\circ} \mathrm{C}$.

Os resíduos de embalagens de Pinus sp. podem ser utilizados satisfatoriamente como matéria-prima para a produção de painéis do tipo aglomerado.

\section{AGRADECIMENTOS}

À FAPEMIG pelo apoio financeiro dos projetos desta linha de pesquisa.

\section{REFERÊNCIAS}

ARAÚJO, S. O. et al. Propriedades de madeiras termorretificadas de Eucalyptus grandis e SP. Scientia Forestalis, v. 40, n. 95, p.327-336, 2012.

ASSOCIAÇÃOBRASILEIRA DENORMAS

TÉCNICAS - ABNT. Chapas de madeira aglomerada. ABNT. NBR 14810-1, 2, 3. mar., 2002.

BOONSTRA, M. J. et al. The effects of a two stage heat treatment process on the properties of particleboard. European Journal of Wood and Wood Products, v.64, n.2, p.157-164, 2006.

BRASIL. LEI No 12.305, DE 2 DE AGOSTO DE 2010. Institui a Política Nacional de Resíduos Sólidos; altera a Lei $\mathrm{n}^{\circ}$ 9.605, de 12 de fevereiro de 1998; e dá outras providências. Diário Oficial da União - Seção 1 - Página 3, 2010.

BYRNE, C. E.; NAGLE, D. C. Carbonization of wood for advanced materials applications. Carbon, v.35, n.2, p.259-266, 1997.

CÉSAR, A. A. S. Estudo da interação adesivo-partícula em painéis OSB (Oriented Strand Board). 2011. 89f. Dissertação (Mestrado em Ciência e Tecnologia da Madeira) Universidade Federal de Lavras, Lavras, 2011.

ESTEVES, B.; GRACA, J.; PEREIRA, H. Extractive composition and summative chemical analysis of thermally treated eucalypt wood.

Holzforschung, v.62, n.3, p.344-351, 2008.

GIRARD, P.; SHAH, N. Developpement of torrefied wood, an alternative to charcoal for reducing deforestation. Technical Series, v.20, p.101-114, 1991.

GOROYIAS, G. J.; HALE, M. D. C. Heat treatment of wood strands for OSB production: effect on the mechanical properties, water absorption and dimensional stability. Cardiff: International Research Group on Wood Protection, 2002. 1p.

HAKKOU, M. et al. Investigation of wood wettability changes during heat treatment on the basis of chemical analysis. Polymer Degradation and Stability, v.89, n.1, p.1-5, 2005. 
IWAKIRI, S. et al. Produção de chapas de madeira aglomerada de cinco espécies de pinus tropicais. Floresta e Ambiente, v.8, n.1, p.137-142, 2001

JENNINGS, J. D. Investigating the surface energy and bond performance of compression densified wood. Dissertation (Master of Science in Wood Science and Forest Products) - Polytechnic Institute and State University, Virginia, 2003.

KELLY, M. W. Critical literature review of relationships between processing parameters and physical properties of particleboard. USDA Forest Service General Technical Reports, FPL, v. 10, p.1-65, 1977.

MENDES, R. F. Efeito do tratamento térmico sobre as propriedades de painéis OSB. 2010. 116f. Dissertação (Mestrado em Recursos Florestais) Universidade de São Paulo - Escola Superior de Agricultura “Luiz de Queiroz”, Piracicaba, 2010.

PAUL, W. et al. Optimising the properties of OSB by a one-step heat pre-treatment process. European Journal of Wood and Wood Products, v.64, n.3, p.227-234, 2006.
PIZZI, A.; MITTAL, K. L.

Handbook of adhesive technology. New York: Marcell Dekker, 1994. 680p.

SERNEK, M.; KAMKE, A.F.; GLASSER, W.G. Comparative analysis of inactivated wood surface. Holzforschung, v.58, n.1, p.22-31, 2004.

STAMM, A. J.; BURR, H. K.; KLINE, A. A. Staybwood - heat-stabilized wood. Industrial \& Engineering Chemistry, v.38, n.6, p.630-634, 1946 .

TJEERDSMA, B. F. et al. Characterization of thermally modified wood: molecular reasons for wood performance improvement. Holz als Roh- und Werkstoff, v.56, p.149-153, 1998.

VERNOIS, M. Heat treatment of wood in Francestate of the art. In: SPECIAL SEMINAR "REVIEW ON HEAT TREATMENTS OF WOOD”, 2001, Paris/France. Annals... Paris: Centre Technique du Bois et de l'Ameublement, 2001.

VITAL, B. R. Métodos de determinação de densidade da madeira. Viçosa, MG: SIF, 1984.21p.

VITAL, B. R.; TRUGILHO, P. F. Variação dimensional e uso da madeira de Eucalyptus. Informe Agropecuário, v.18, n.186, p.57-61, 1997. 